\title{
Mechanically induced silyl ester cleavage under acidic conditions investigated by AFM-based single-molecule force spectroscopy in the force-ramp mode
}

\author{
Sebastian W. Schmidt, ${ }^{\text {abc }}$ Michael F. Pill, ${ }^{\text {abc }}$ Alfred Kersch, ${ }^{a}$ \\ Hauke Clausen-Schaumann ${ }^{\text {ac }}$ and Martin K. Beyer ${ }^{\star b d}$
}

Received 4th December 2013, Accepted 17th January 2014

DOI: 10.1039/c3fd00119a

AFM-based dynamic single-molecule force spectroscopy was used to stretch carboxymethylated amylose (CMA) polymers, which have been covalently tethered between a silanized glass substrate and a silanized AFM tip via acid-catalyzed ester condensation at $\mathrm{pH}$ 2.0. Rupture forces were measured as a function of temperature and force loading rate in the force-ramp mode. The data exhibit significant statistical scattering, which is fitted with a maximum likelihood estimation (MLE) algorithm. Bond rupture is described with a Morse potential based Arrhenius kinetics model. The fit yields a bond dissociation energy $D_{\mathrm{e}}=35 \mathrm{~kJ} \mathrm{~mol}^{-1}$ and an Arrhenius prefactor $A=6.6 \times 10^{4} \mathrm{~s}^{-1}$. The bond dissociation energy is consistent with previous experiments under identical conditions, where the force-clamp mode was employed. However, the bi-exponential decay kinetics, which the force-clamp results unambiguously revealed, are not evident in the force-ramp data. While it is possible to fit the force-ramp data with a bi-exponential model, the fit parameters differ from the force-clamp experiments. Overall, single-molecule force spectroscopy in the force-ramp mode yields data whose information content is more limited than force-clamp data. It may, however, still be necessary and advantageous to perform force-ramp experiments. The number of successful events is often higher in the force-ramp mode, and competing reaction pathways may make force-clamp experiments impossible.

\footnotetext{
${ }^{a}$ Munich University of Applied Sciences, Department of Applied Natural Sciences and Mechatronics, Lothstr. 34, 80335 Munich, Germany. E-mail: clausen-schaumann@hm.edu; alfred.kersch@hm.edu; Fax: +49891265 1603; Tel: +498912651615

${ }^{b}$ Institut für Physikalische Chemie, Christian-Albrechts-Universität zu Kiel, Olshausenstrasse 40, 24098 Kiel, Germany

${ }^{c}$ Center for NanoScience (CeNS), Geschwister-Scholl-Platz 1, 80539 Munich, Germany

${ }^{d}$ Institut für Ionenphysik und Angewandte Physik, Leopold-Franzens-Universität Innsbruck, Technikerstrasse 25, 6020 Innsbruck, Austria. E-mail: martin.beyer@uibk.ac.at; Fax: +43 512507 2932; Tel: +43 51250752680
} 


\section{Introduction}

Covalent mechanochemistry has been rapidly developing over the last decade. ${ }^{1-3}$ Quantitative theory has matured, with a variety of approaches available for a wide range of problems, like COnstrained Geometry simulates External Force (COGEF), ${ }^{4}$ External Force Explicitly Included (EFEI), ${ }^{5}$ Force Modified Potential Energy Surfaces (FMPES) ${ }^{6}$ including approximate models based on numerical expansion of the FMPES. ${ }^{7}$ For quantitative experiments, the instrument of choice is the atomic force microscope (AFM). Since its introduction in $1994,{ }^{8}$ AFM based single-molecule force spectroscopy (SMFS) has been successfully applied in a versatile manner from the investigation of receptor ligand interactions, ${ }^{\mathbf{8 - 1 0}}$ protein folding, ${ }^{11-15}$ adhesion forces and polymer elasticity, ${ }^{16-23}$ to studies addressing the mechanical properties of covalent bonds., ${ }^{\mathbf{1 , 2 4 - 2 9}}$

In early SMFS surveys, intermolecular and intramolecular forces were extracted independently of the involved mechanical transient by approximating a constant force-loading rate. ${ }^{8,24}$ By expanding this strategy to the dynamic SMFS approach, where the force-loading rate is varied over an appropriate range, a typically nearly linear relationship between binding strength and the logarithm of the force-loading rate can be found. Thus, the structural and kinetic parameters of the underlying process become accessible..$^{13,30-32}$

In order to obtain the kinetic parameters from such dynamic SMFS experiments, different theoretical models have been developed, ${ }^{4,33-36}$ all considering bond rupture as a thermally activated process, where the activation barrier is lowered by the mechanical energy stored in the deflected cantilever. A general description of such a thermally activated rupture process is provided by Arrhenius type kinetics, with a force-dependent activation energy $E_{\mathrm{a}}$, where the bond lifetime $\tau=1 / k^{\text {off }}$ is given by $k^{\text {off }}=A \exp \left[-E_{\mathrm{a}}(f) / k_{\mathrm{B}} T\right]$.

For the theoretical description of covalent bonds under mechanical load, an Arrhenius kinetics model combined with a Morse potential has been widely used as an approximation. ${ }^{4,35,37,38}$ With the force-dependent deformation of the Morse potential, which enters the force-dependent activation energy in the Arrhenius equation, bond rupture probabilities can be numerically calculated and the dynamics, as well as the structural parameters of these single bond rupture events, can be extracted from the experimental data. ${ }^{26}$

A more recent approach in the realm of AFM-based SFMS is the force-clamp SMFS method, where an individually coupled molecule is stretched with a defined force, and retained at this force until bond scission occurs. The recorded force versus time plot yields the bond survival time as a function of the clamp force and temperature. A large number of statistically distributed bond survival times provides the complete unimolecular kinetics of the bond dissociation event, and allows for distinguishing multiexponential decay kinetics. ${ }^{39}$ In contrast to dynamic SMFS, the force-clamp SMFS technique provides direct access to the reaction kinetics of mechanically activated processes on the molecular level. ${ }^{12,14,15,40-43}$ By analyzing the recorded data with an Arrhenius kinetics model with a force-dependent activation barrier, the measured reaction rate constants can be used directly to calculate force and temperature-independent parameters, like the activation energy, the Arrhenius pre-factor and the width of the binding potential. 
In a recent study, ${ }^{39}$ we took advantage of force-clamp SMFS to record the force and temperature-dependent bond survival times of carboxymethylated amylose (CMA) covalently tethered to silane functionalized silicon oxide surfaces via acidcatalyzed ester condensation at $\mathrm{pH} 2.0{ }^{44}$ The conducted experiments revealed biexponential rupture kinetics, which have been rationalized with the silyl ester hydrolysis depending on the moieties at the silicon atom under acidic conditions.

Here, we have performed temperature-dependent dynamic SMFS to stretch individual CMA polymers under similar conditions in order to determine the structural parameters of the binding potential, i.e. the bond dissociation energy $D_{\mathrm{e}}$, the parameter $\beta^{-1}$, which is proportional to the potential width, and the Arrhenius pre-factor $A$. Comparing the obtained data with the results from forceclamp SMFS ${ }^{39}$ reaffirm the assumption of a silyl ester hydrolysis mechanism. The advantages and disadvantages of force-clamp SMFS and the more widely used dynamic SMFS method are discussed.

\section{Experimental procedure and data analysis}

\subsection{Materials}

Carboxymethylated amylose (CMA), $N^{1}$-[3-(trimethoxysilyl)-propyl]diethylene-triamine (DETA) and phosphate buffered saline (PBS; buffer composed of $0.137 \mathrm{M}$ $\mathrm{NaCl}, 0.010 \mathrm{M} \mathrm{Na}_{2} \mathrm{HPO}_{4}, 0.003 \mathrm{M} \mathrm{KCl}$, and $0.002 \mathrm{M} \mathrm{KH}_{2} \mathrm{PO}_{4}, \mathrm{pH} 7.4$ at $\mathrm{T}=25^{\circ} \mathrm{C}$ ) were purchased from Sigma-Aldrich (Deisenhofen, Germany). Hydrochloric acid (32\% GR for analysis), acetic acid (99-100\% for synthesis) and ethanol (absolute GR for analysis) were obtained from Merck (Darmstadt, Germany). All experiments were performed with silicon nitride AFM cantilevers with a nominal force constant between 10 and $20 \mathrm{mNm}^{-1}$ (MLCT-AU, Veeco Instruments GmbH, Mannheim, Germany). Glass microscope slides were obtained from Menzel (Braunschweig, Germany).

\subsection{Experimental setup}

The sample preparation was performed as previously described.9,44 Glass microscope slides were immersed in diluted hydrochloric acid for $120 \mathrm{~min}$, sonicated in a cleaning solution for $60 \mathrm{~min}$, and rinsed with water three times. The silicon nitride AFM cantilevers were irradiated with UV light for $60 \mathrm{~min}$ and immersed in ethanol. The glass slides and cantilever surfaces were functionalized with DETA by immersion for $60 \mathrm{~min}$ in a $10: 1$ ethanol : water mixture, acidified with acetic acid to $\mathrm{pH} 4.5-5.5$, with a DETA content of $2 \mathrm{vol} \%$. After rinsing with ethanol, the slides and cantilevers were cured at $110{ }^{\circ} \mathrm{C}$ for $20 \mathrm{~min}$. Prior to individual SMFS experiments, CMA was suspended in PBS titrated to $\mathrm{pH} 2.0$ with diluted hydrochloric acid and transferred to the functionalized glass substrate, followed by thorough rinsing. The slide was then mounted on the AFM stage and covered with PBS buffer, which was also acidified to $\mathrm{pH}$ 2.0. Previous experiments with DETA and three other organosilanes under identical experimental conditions showed that CMA is linked to DETA via an acid-catalyzed ester condensation. ${ }^{\mathbf{4 4}}$

In order to perform temperature-dependent SMFS experiments, a thermostat (CF30 Kryo-Kompakt-Thermostat, Julabo Labortechnik GmbH, Seelbach, Germany) was connected with a fluid cooler (FLKU 140 G 200, Fischer Elektronik 
GmbH, Lüdenscheid, Germany) serving as slide holder, as described elsewhere. ${ }^{26}$ After a constant temperature of the solution at the stage was obtained, the SMFS experiments were conducted using an atomic force microscope (NanoWizard, JPK Instruments, Berlin, Germany) in the force spectroscopy mode. The AFM tip was approached to the glass substrate with covalently coupled CMA, and force $v$. distance curves were recorded. The maximum contact force between the AFM tip and the substrate prior to recording a force $v s$. distance curve was kept below $0.3 \mathrm{nN}$, and the contact time between the AFM tip and the substrate was kept between $0.5 \mathrm{~s}$ and $1.0 \mathrm{~s}$.

\subsection{Data analysis}

The experimental results were analyzed in a parallel fit procedure using a Morse potential based Arrhenius rate equation as previously described in detail. ${ }^{26}$ Forceloading rate-dependent bond rupture distributions were numerically calculated and optimized by varying the parameters $D_{\mathrm{e}}, f_{\max }$ and $A$. In order to account for the exact shape of the bond rupture distributions, parameter optimization was performed with the maximum likelihood estimator method. ${ }^{45}$ For reasons of better comparability with the results obtained from the force-clamp SMFS experiments, the Arrhenius pre-factor $A$ was treated as a temperature-independent parameter and the potential width $\beta^{-1}$ was calculated via $\beta=2 f_{\max } / D_{\mathrm{e}}{ }^{4}$ The parameter errors were calculated as previously described ${ }^{39}$ with the MINOS algorithm, which is included in the MINUIT analysis tool. ${ }^{46}$ The optimum parameters obtained from the MLE procedure were varied until the maximum of the log-likelihood function was reduced by the value 0.5 , which defines the confidence interval of $68.3 \% .^{47}$

\section{Results and discussion}

Scheme 1 displays a single CMA polymer tethered between a glass substrate and AFM tip via silyl ester bonds. These bonds are formed under acidic conditions, where a proton catalyzed condensation reaction takes place between the carboxyl groups in the CMA and unreacted hydroxyl groups on the glass surface or on the silane surface anchor. ${ }^{38}$

When the cantilever retracts at a constant velocity from the surface, the individually picked up CMA molecule is stretched until the connection between the AFM tip and substrate is lost. One can obtain the bond rupture force as well as the force-loading rate from the resulting force-extension curve. ${ }^{27}$ Varying the retract velocity allows the coverage of a wide range of force-loading rates $\dot{f}$. If the forceloading rate is systematically varied, scatter plots can be obtained as shown in Figure 1, where the bond rupture force is plotted versus the force-loading rate.

In order to extract the kinetic parameters from the measured data recorded at five temperatures between $282 \mathrm{~K}$ and $320 \mathrm{~K}$, the five data sets were analyzed with a parallel fit procedure using an Arrhenius kinetics model in combination with a Morse potential, as previously described. ${ }^{27}$ In brief, the force-loading ratedependent rupture force probability distributions were optimized for all five temperatures using the maximum likelihood estimation (MLE) method.

The rupture force distributions (green shaded areas) shown in Figure 1 were calculated with the obtained fit parameters $D_{\mathrm{e}}=35 \mathrm{~kJ} \mathrm{~mol}^{-1}, \beta^{-1}=0.061 \AA$, and 


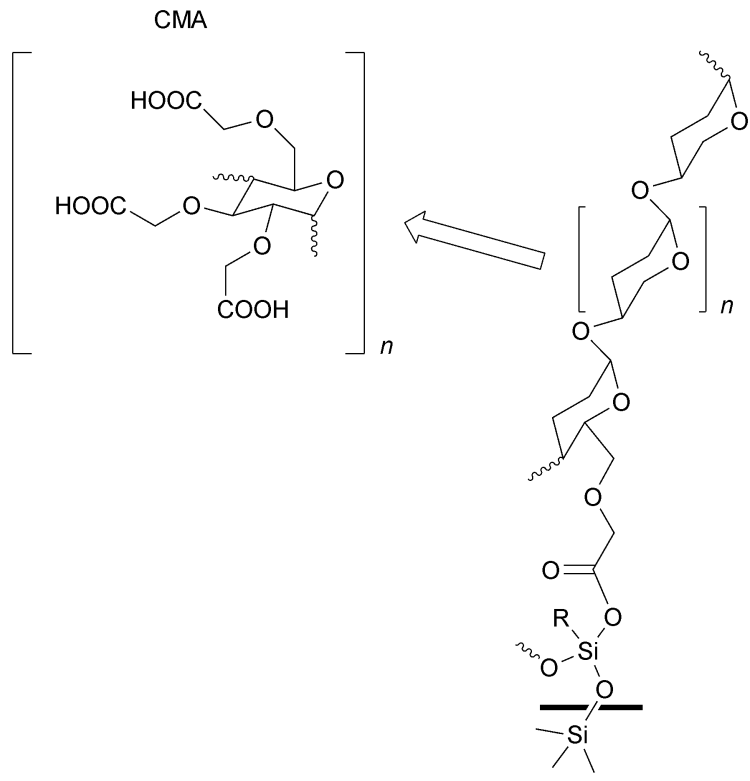

Scheme 1 An individual CMA polymer coupled between a silanized AFM tip and a silanized substrate. Under acidic conditions, the carboxyl groups of the CMA and free hydroxyl groups, which are present on free silanol groups in the silane anchor, undergo protoncatalyzed ester condensation.

the corresponding Arrhenius pre-factor $A=6.6 \times 10^{4} \mathrm{~s}^{-1}$. The extracted parameters are summarized in Table 1 and compared to values as determined from experiments conducted under similar conditions using force-clamp SMFS.

The bond dissociation energy $D_{\mathrm{e}}=34.7 \mathrm{~kJ} \mathrm{~mol}^{-1}$ as well as the Arrhenius prefactor $A=6.6 \times 10^{4} \mathrm{~s}^{-1}$ determined from dynamic SMFS are almost identical with the ones obtained for the slow process from the force-clamp SMFS experiments, pointing towards the observation of an identical bond rupture processes in both the dynamic and the force-clamp SMFS approach. This serves as a first hint, that the two AFM-based techniques provide consistent kinetic parameters on the single-molecule level, which clearly demonstrates the reliability of the two SMFS strategies. However, the parameters from the dynamic SMFS correspond to the parameters for the slow process and slightly differ from the values for the fast process, which become evident under force-clamp conditions. Together with $\beta^{-1}$ $=0.061 \AA$, which is found to be twice as large as the values extracted from the force-clamp SMFS experiments, the observed variations may be explained by systemic differences concerning data extraction and analysis.

The most obvious difference between the two experimental approaches is given by the fact that experiments conducted by means of dynamic SMFS do not allow for the distinction of multi-exponential kinetics, i.e. different subsets of decays typically remain undetected. This can be attributed to the force-loading rate dependence in the case of dynamic SMFS: while the force-clamp SMFS experiments directly yield reaction rate constant(s) $k(f, T)$, which depend on the clamp force and temperature, the reaction rate constant, which determines the data obtained from dynamic SMFS experiments, additionally depends on the 


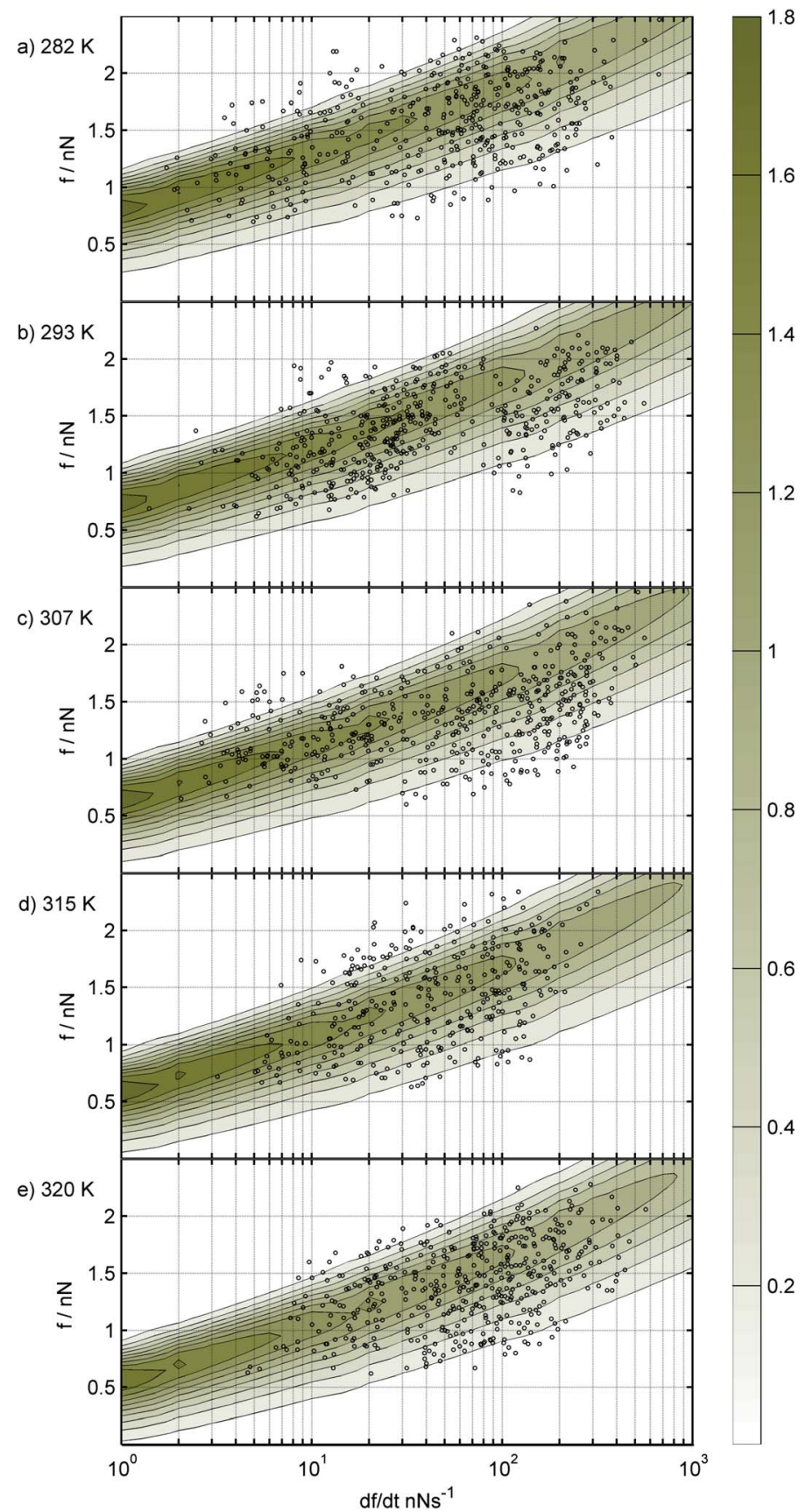

Fig. 1 The bond rupture forces $f v s$. the force loading rates $d f / d t$ of more than 2150 single molecule rupture events at $282 \mathrm{~K}(\mathrm{a}), 293 \mathrm{~K}$ (b), $307 \mathrm{~K}$ (c), $315 \mathrm{~K}$ (d) and $320 \mathrm{~K}(\mathrm{e})$. Every data point corresponds to one individual rupture event. The green shaded areas show the expected distribution calculated with the fit parameters $D_{\mathrm{e}}=34.7 \mathrm{~kJ} \mathrm{~mol}^{-1}, \beta^{-1}=0.061 \AA$, and an Arrhenius pre-factor of $A=6.6 \times 10^{4} \mathrm{~s}^{-1}$. 
Table 1 The parameters extracted from experimental data using an Arrhenius kinetics model in combination with a Morse potential

\begin{tabular}{|c|c|c|c|c|c|}
\hline \multirow[b]{2}{*}{ Parameter $^{a}$} & \multirow{2}{*}{$\begin{array}{l}\text { Dynamic } \\
\text { SMFS }^{b d}\end{array}$} & \multicolumn{2}{|c|}{ Dynamic SMFS $^{c d}$} & \multicolumn{2}{|c|}{ Force-clamp SMFS ${ }^{e f}$} \\
\hline & & slow & fast & slow & fast \\
\hline$D_{\mathrm{e}} / \mathrm{kJ} \mathrm{mol}^{-1}$ & $34.7 \pm 1.2$ & $54.9 \pm 2.3$ & $36.9 \pm 1.7$ & $39.2 \pm 5.7$ & $39.5 \pm 5.1$ \\
\hline$\beta^{-1} / \AA$ & $0.061 \pm 0.023$ & $0.117 \pm 0.005$ & $0.098 \pm 0.011$ & $0.033 \pm 0.0035$ & $0.033 \pm 0.020$ \\
\hline$A / \mathrm{s}^{-1}$ & $\begin{array}{l}6.6 \pm 0.2 \\
\times 10^{4}\end{array}$ & $\begin{array}{l}2.0 \pm 1.0 \\
\times 10^{5}\end{array}$ & $\begin{array}{l}2.5 \pm 1.1 \\
\times 10^{4}\end{array}$ & $\begin{array}{l}6.0 \pm 1.9 \\
\times 10^{4}\end{array}$ & $\begin{array}{l}2.0 \pm 0.5 \\
\times 10^{6}\end{array}$ \\
\hline
\end{tabular}

${ }^{a}$ Free parameters from the Morse potential-based Arrhenius kinetics model. ${ }^{b}$ Values extracted from the global fit to the experimental data shown in Fig. 1a-e. ${ }^{c}$ Experimental data shown in Fig. 2a-e with a bi-exponential global fit where the slow process is contributing $32 \%$ and the fast one $68 \%$. ${ }^{d}$ Errors were obtained by calculating the standard-deviation from a numerically calculated hessian matrix. ${ }^{e}$ Results from forceclamp SMFS experiments under similar conditions, ${ }^{39}$ where a bi-exponential behaviour was found. According to the bi-exponential fit the overall process is subdivided into two fractions, where the slow process contributes $28 \%$ and the fast one $72 \%$. ${ }^{f}$ The parameters were calculated with the MLE procedure as previously described. ${ }^{26}$ The parameter errors correspond to one-standard-deviation errors as calculated according to the MINOS algorithm as part of the MINUIT analysis tool. ${ }^{47}$

force-loading rate, i.e. $k(f, \dot{f}, T)$. As a consequence, with the force-loading rate as an additional variable, the experimental data from dynamic SMFS investigations can be described by an arbitrary number of reaction rate constants, which makes a clear distinction of multiple decays practically impossible. Consequently, if the values from the dynamic SMFS summarized in Table 1 actually result from a biexponential decay, one has to work with the assumption that the dynamic SMFS parameters represent the optimum compromise between the parameters extracted from the two processes using force-clamp SMFS. However, fitting a data set, which apparently consists of two superimposed data entities, with one single parameter set, might be expected to be accompanied by higher parameter errors due to the influence of larger data scattering. As can be seen from Table 1, the contrary is the case here.

The fact that the parameter errors are even larger for the values extracted from force-clamp SMFS, can be ascribed to the assumption of an inconsistent bond rupture distribution in order to analyze the data recorded with dynamic SMFS. Generally, the MLE-based parameter optimization aims for a bond rupture distribution that makes the observed data most probable, i.e. the most likely probability for the ensemble of data point is calculated by varying the unknown fit parameters. ${ }^{45}$ The latter is crucial in order to obtain consistent parameter estimates for the observed data. By analyzing two sub-processes with one single set of bond rupture distributions, parameters corresponding to the best fit are indeed found, but the underlying model assumption is inconsistent with the observations from force-clamp SMFS.

As a consequence, in order to be able to directly compare the parameters from dynamic SMFS and force-clamp SMFS, modelling the data shown in Figure 1 with two bond rupture distribution sets is inevitable. Figure 2 shows the results of such a fit, where the branching ratio between the two processes has been treated as a fit parameter. As expected, with the higher flexibility of the fit, the likelihood 


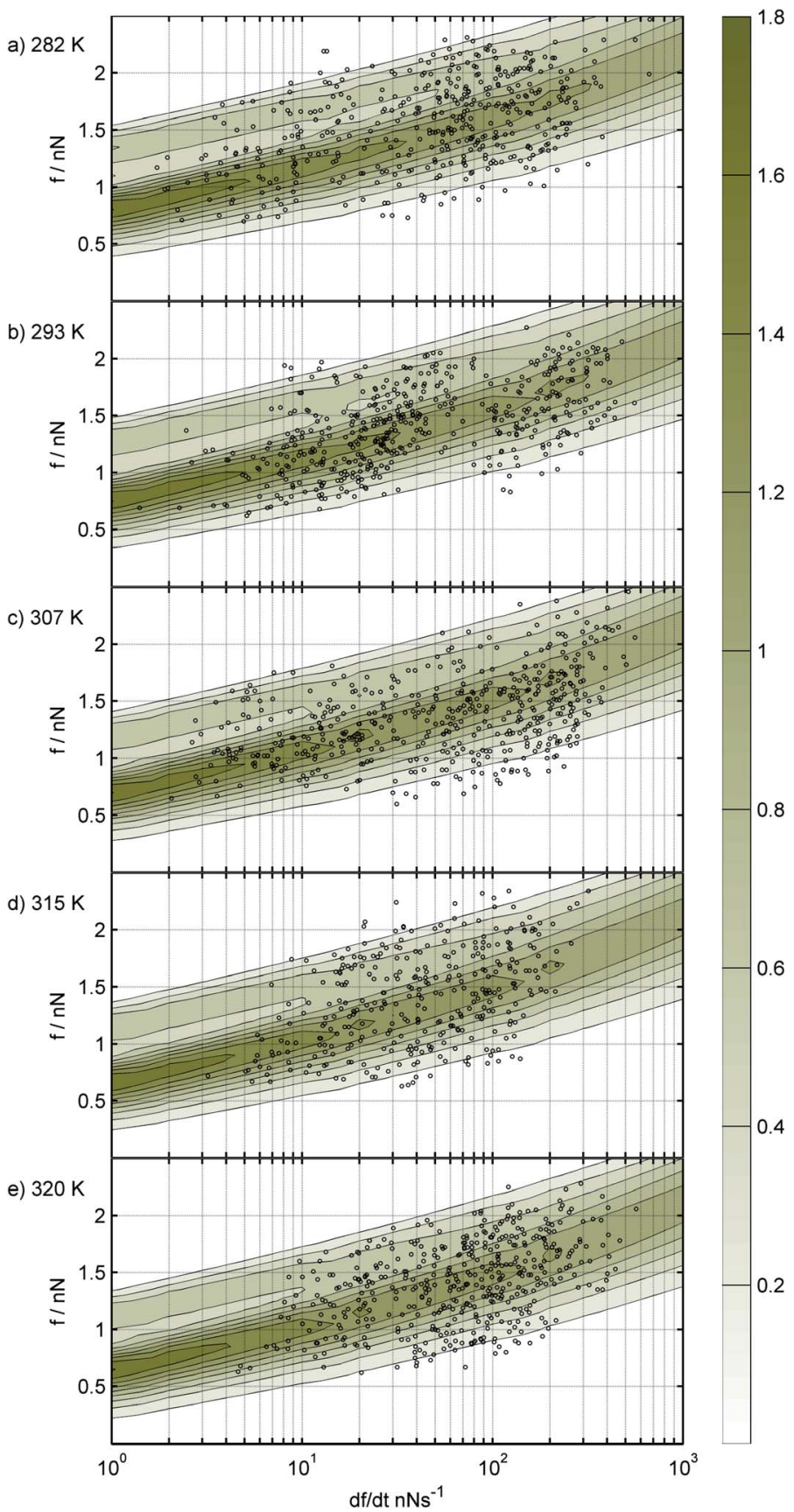

Fig. 2 Here the green shaded areas show the expected distribution calculated with the biexponential fit parameters for the slow process of $D_{\mathrm{e}, \mathrm{s}}=54.9 \mathrm{~kJ} \mathrm{~mol}^{-1}, \beta^{-1} \mathrm{~s}=0.117 \AA$, and an Arrhenius pre-factor of $A_{\mathrm{s}}=2.0 \times 10^{5} \mathrm{~s}^{-1}$, for the fast process of $D_{\mathrm{e}, \mathrm{f}}=36.9 \mathrm{~kJ} \mathrm{~mol}^{-1}$, $\beta^{-1}{ }_{f}=0.098 \AA$, and an Arrhenius pre-factor of $A_{f}=2.5 \times 10^{4} \mathrm{~s}^{-1}$. The two processes are combined with a weight factor of 0.32 for the slow and 0.68 for the fast process. 
function improves significantly. This alone is not unambiguous proof that the biexponential fit is more correct. The fact, however, that the branching ratio between the slow and the fast process is similar to the fit of the force-clamp results indicates that the two data sets are consistent, and that the key idea of a biexponential behaviour is correct. The fit parameters for the slow process are $D_{\mathrm{e}, \mathrm{s}}=$ $54.9 \mathrm{~kJ} \mathrm{~mol}^{-1}, \beta_{\mathrm{s}}^{-1}=0.117 \AA$, and an Arrhenius pre-factor of $A_{\mathrm{s}}=2.0 \times 10^{5} \mathrm{~s}^{-1}$, for the fast process they are $D_{\mathrm{e}, \mathrm{f}}=36.9 \mathrm{~kJ} \mathrm{~mol}^{-1}, \beta^{-1} \mathrm{f}=0.098 \AA$, and an Arrhenius pre-factor of $A_{\mathrm{f}}=2.5 \times 10^{4} \mathrm{~s}^{-1}$. The two processes contribute with a weight factor of 0.32 for the slow and 0.68 for the fast process. The results of the force-ramp and force-clamp fits are summarized in Table 1 to facilitate comparison.

The parameters of the dominant fast process, in particular the dissociation energy $D_{\mathrm{e}}$, compare favorably with the single-exponential fit. The force-clamp results deviate slightly for the fast process, where a higher dissociation energy is compensated by a higher pre-factor $A$. Significantly different, however, is the dissociation energy for the slow process. With $54.9 \mathrm{~kJ} \mathrm{~mol}^{-1}$, it is $15.7 \mathrm{~kJ} \mathrm{~mol}^{-1}$ higher than the corresponding value from the force-clamp fit, and lies well outside the numerical error limits. This may in part be due to the smaller contribution of the slow process. More likely, however, is a systematic problem. The Morse potential based Arrhenius kinetics model, initially developed for the homolytic rupture of a covalent bond in the polymer backbone, is not a very good approximation for the potential energy surface of bond hydrolysis. ${ }^{48}$ In the absence of a better model, it is still worthwhile to conduct these fits. A fully consistent picture, with quantitatively reliable results, however, will only emerge when a realistic description of the potential energy surface of the reaction is available and implemented into the fit procedure.

\section{Conclusions}

In the present study, we have identified structural and kinetic parameters for the mechanically induced rupture of individual covalent bonds using temperaturedependent dynamic SMFS at pH 2.0. Except for the slow process of the bi-exponential fit, the kinetic parameters agree well with the ones extracted from forceclamp SMFS experiments performed under acidic conditions. This clearly indicates the observation of the analogous bond rupture mechanism. The results corroborate that silyl ester hydrolysis is observed at low $\mathrm{pH}$. The consistent results first of all demonstrate the functional equivalence of the two AFM-based singlemolecule approaches. However, unambiguous identification of a bi-exponential behaviour, which has been clearly evident in force-clamp SMFS, appeared to be impossible with the dynamic SMFS technique. As a consequence, analyzing the data presented here on the basis of one single bond rupture mechanism yields parameters, which are directly comparable to the parameters from force-clamp SMFS with reservations only. When the analysis model is extended to account for two isomeric decays, dynamic SMFS experiments yield results that deviate from the force-clamp experiments. This may in part be attributed to the low number of recorded bond rupture events per force-loading rate representing a statistically weak data basis for the calculation of two weighted bond rupture distributions. Another limitation is the use of a Morse potential to describe the potential energy surface of a hydrolysis reaction. 
Experiments conducted in the force-clamp SMFS mode with the clamp force as the controllable experimental parameter, allow for the direct observation of multiple isomeric decays. This substantial advantage considerably extends the capabilities of AFM-based SMFS methods to the extraction of kinetic parameters from multi-exponential processes on the single-molecule level, which are hardly accessible with the widely used dynamic SMFS approach. Force-ramp experiments will still be useful in cases where the number of successful events is too low in the force-clamp mode, and where the force-distance curve yields additional information.

\section{Acknowledgements}

S.W.S., M.F.P. and H.C.-S. gratefully acknowledge financial support of the German Excellence Initiative via the "Nanosystems Initiative Munich (NIM)". M.K.B. gratefully acknowledges funding from the Sonderforschungsbereich 677 "Function by Switching" supported by the Deutsche Forschungsgemeinschaft (DFG).

\section{References}

1 M. K. Beyer and H. Clausen-Schaumann, Chem. Rev., 2005, 105, 2921.

2 M. M. Caruso, D. A. Davis, Q. Shen, S. A. Odom, N. R. Sottos, S. R. White and J. S. Moore, Chem. Rev., 2009, 109, 5755.

3 J. Ribas-Arino and D. Marx, Chem. Rev., 2012, 112, 5412.

4 M. K. Beyer, J. Chem. Phys., 2000, 112, 7307.

5 J. Ribas-Arino, M. Shiga and D. Marx, Angew. Chem., Int. Ed., 2009, 48, 4190.

6 M. T. Ong, J. Leiding, H. Tao, A. M. Virshup and T. J. Martínez, J. Am. Chem. Soc., 2009, 131, 6377.

7 A. Bailey and N. J. Mosey, J. Chem. Phys., 2012, 136, 44102.

8 E. L. Florin, V. T. Moy and H. E. Gaub, Science, 1994, 264, 415.

9 P. Hinterdorfer, W. Baumgartner, H. J. Gruber, K. Schilcher and H. Schindler, Proc. Natl. Acad. Sci. U. S. A., 1996, 93, 3477.

10 F. Schwesinger, R. Ros, T. Strunz, D. Anselmetti, H.-J. Guntherodt, A. Honegger, L. Jermutus, L. Tiefenauer and A. Pluckthun, Proc. Natl. Acad. Sci. U. S. A., 2000, 97, 9972.

11 M. Rief, M. Gautel, F. Oesterhelt, J. M. Fernandez and H. E. Gaub, Science, 1997, 276, 1109.

12 A. F. Oberhauser, P. K. Hansma, M. Carrion-Vazquez and J. M. Fernandez, Proc. Natl. Acad. Sci. U. S. A., 2001, 98, 468.

13 M. Rief and H. Grubmüller, ChemPhysChem, 2002, 3, 255.

14 J. M. Fernandez and H. B. Li, Science, 2004, 303, 1674.

15 B. Bullard, T. Garcia, V. Benes, M. C. Leake, W. A. Linke and A. F. Oberhauser, Proc. Natl. Acad. Sci. U. S. A., 2006, 103, 4451.

16 M. Rief, F. Oesterhelt, B. Heymann and H. E. Gaub, Science, 1997, 275, 1295.

17 P. E. Marszalek, A. F. Oberhauser, Y. P. Pang and J. M. Fernandez, Nature, 1998, 396, 661.

18 T. Hugel, M. Grosholz, H. Clausen-Schaumann, A. Pfau, H. Gaub and M. Seitz, Macromolecules, 2001, 34, 1039. 
19 Z. Lu, W. Nowak, G. Lee, P. E. Marszalek and W. Yang, J. Am. Chem. Soc., 2004, 126, 9033.

20 E. Thormann, D. R. Evans and V. S. J. Craig, Macromolecules, 2006, 39, 6180.

21 Q. Zhang and P. E. Marszalek, Polymer, 2006, 47, 2526.

22 M. I. Giannotti and G. J. Vancso, ChemPhysChem, 2007, 8, 2290.

23 K. Liu, Y. Song, W. Feng, N. Liu, W. Zhang and X. Zhang, J. Am. Chem. Soc., 2011, 133, 3226.

24 M. Grandbois, M. Beyer, M. Rief, H. Clausen-Schaumann and H. E. Gaub, Science, 1999, 283, 1727.

25 P. Schwaderer, E. Funk, F. Achenbach, J. Weis, C. Bräuchle and J. Michaelis, Langmuir, 2008, 24, 1343.

26 S. W. Schmidt, A. Kersch, M. K. Beyer and H. Clausen-Schaumann, Phys. Chem. Chem. Phys., 2011, 13, 5994.

27 S. W. Schmidt, M. K. Beyer and H. Clausen-Schaumann, J. Am. Chem. Soc., 2008, 130, 3664.

28 J. Liang and J. M. Fernández, J. Am. Chem. Soc., 2011, 133, 3528.

29 H. M. Klukovich, T. B. Kouznetsova, Z. S. Kean, J. M. Lenhardt and S. L. Craig, Nat. Chem., 2012, 5, 110.

30 R. Merkel, P. Nassoy, A. Leung, K. Ritchie and E. Evans, Nature, 1999, 397, 50.

31 B. Heymann and H. Grubmüller, Phys. Rev. Lett., 2000, 84, 6126.

32 T. Hugel and M. Seitz, Macromol. Rapid Commun., 2001, 22, 989.

33 G. I. Bell, Science, 1978, 200, 618.

34 E. Evans and K. Ritchie, Biophys. J., 1997, 72, 1541.

35 F. Hanke and H. J. Kreuzer, Phys. Rev. E: Stat., Nonlinear, Soft Matter Phys., 2006, 74, 31909.

36 O. Dudko, G. Hummer and A. Szabo, Phys. Rev. Lett., 2006, 96, 108101.

37 W. Kauzmann and H. Eyring, J. Am. Chem. Soc., 1940, 62, 3113.

38 M. F. Iozzi, T. Helgaker and E. Uggerud, Mol. Phys., 2009, 107, 2537.

39 S. W. Schmidt, P. Filippov, A. Kersch, M. K. Beyer and H. Clausen-Schaumann, ACS Nano, 2012, 6, 1314.

40 A. P. Wiita, S. R. K. Ainavarapu, H. H. Huang and J. M. Fernandez, Proc. Natl. Acad. Sci. U. S. A., 2006, 103, 7222.

41 S. R. K. Ainavarapu, A. P. Wiita, L. Dougan, E. Uggerud and J. M. Fernandez, J. Am. Chem. Soc., 2008, 130, 6479.

42 R. Szoszkiewicz, S. R. K. Ainavarapu, A. P. Wiita, R. Perez-Jimenez, J. M. Sanchez-Ruiz and J. M. Fernandez, Langmuir, 2008, 24, 1356.

43 J. Liang and J. M. Fernández, ACS Nano, 2009, 3, 1628.

44 S. W. Schmidt, T. Christ, C. Glockner, M. K. Beyer and H. Clausen-Schaumann, Langmuir, 2010, 26, 15333.

45 S. Brandt, Data analysis. Statistical and computational methods for scientists and engineers, Springer, New York, 3rd edn., 1999.

46 F. James and M. Roos, Comp. Phys. Comm., 1975, 10, 343.

47 CN/ASD Group, MINUIT - Users Guide. nProgram Library D506, CERN, 1993.

48 M. F. Pill, S. W. Schmidt, M. K. Beyer, H. Clausen-Schaumann and A. Kersch, J. Chem. Phys., 2014, 140, 044321. 